\title{
Specialized advertising and price competition in vertically differentiated markets
}

\author{
Lola Esteban • José M. Hernández
}

Received: 30 October 2009 / Accepted: 8 October 2010 / Published online: 2 November 2010 (C) The Author(s) 2010. This article is published with open access at Springerlink.com

\begin{abstract}
This paper studies how the emergence of specialized communication media focused on both high quality contents and high quality advertised products, affects the functioning of a vertically differentiated market. To that end, we formulate a simultaneous game of pricing and targeted advertising with two firms producing different levels of quality. We find that the transition from uniform advertising to targeted advertising can turn a pure vertically differentiated market into a hybrid market which incorporates some features of a monopoly, thus changing the pattern of price competition between the firms. In particular, we show that (1) compared to uniform advertising, targeting leads both firms to always charge higher prices, (2) the increase in prices is more intense in highly competitive (low differentiated) markets, (3) the expected price of the low-quality firm is non-monotonic with the degree of product differentiation, and (4) the low-quality product may be sold at a higher expected price than the high-quality product. We also show that a progressive growth of specialized advertising vehicles leads to a further increase in prices. In addition, more specialized targeting may raise price competition, so firms may find it optimal to use low specialized targeting.
\end{abstract}

Keywords Informative advertising - Targeting · Vertical differentiation · Price dispersion

\footnotetext{
The authors would like to thank José Luis Moraga-Gonzalez and two anonymous referees for their constructive comments and suggestions. Financial support from both FEDER and the Spanish Government, through Grant ECO2008-05024/ECON, is gratefully acknowledge.
}

L. Esteban · J. M. Hernández $(\varangle)$

Departamento de Análisis Económico, Facultad de Económicas,

University of Zaragoza, Gran Vía 2, 50.005 Saragossa, Spain

e-mail: hernande@unizar.es 
JEL Classification $\quad \mathrm{D} 43 \cdot \mathrm{L} 13 \cdot \mathrm{M} 37$

\section{Introduction}

The way in which firms promote their products has undergone significant change in recent years. Traditionally, sellers have used the mass media to reach their potential customers and marketing managers have been concerned with how to reduce, to the greatest possible extent, advertising wastage, that is, exposing consumers who are not interested in their products to the advertising campaign. However, as a result of both the progressive fragmentation of traditional media (radio, TV, etc.) and the emergence of new and highly specialized communication channels (the Internet, cable TV, specialized press, etc.), nowadays advertisers have the possibility of targeting their ads on particular segments of the potential demand, which may change the pattern of price competition between firms. Against this background, it is important to determine how the strategic use of targeted advertising can affect the functioning of a market, and this paper offers a first attempt at analyzing this issue in the context of vertically differentiated products.

To address this task, we formulate a price competition model where two firms use informative advertising to promote the sale of goods with different levels of quality. Consumers, who are heterogeneous in their taste for quality, are unaware of the existence of the goods, and sellers can inform them of their existence, price and product specifications by using either uniform advertising, which reaches the whole potential market, or specialized advertising, which targets the ads on a particular segment of the market. In a model of targeting, the fundamental issue is on which segment of the market the specialized media concentrate the ads, i.e. how the targeting technology relates to the demand for the products. In this regard, we note that (1) the media industry has recently produced a huge amount of highly specialized communication channels which reach particular audiences with one or more common interests, and (2) these selective segments of potential consumers tend to have a relatively high valuation of the products advertised in these media. This means that firms operating in a vertically differentiated market have a range of advertising outlets available with different degrees of specialization, and that this specialization is related to consumers' valuations of the advertised product qualities. In particular, we observe that a highly specialized interest medium (like a thematic magazine, radio or cable TV) usually offers high-quality contents to a selective segment of consumers, who are often more inclined to consume the high-quality products which are typically advertised in this type of communication vehicle. Further, in the same product market, sellers can frequently use a less specialized (or general interest) medium, which usually has a wider reach (often including consumers with both a high and a low valuation of quality) and also promotes lower quality products. This pattern of media specialization, which Esteban et al. $(2001,2006)$ call "nested", is quite frequent, for example, in the magazine industry, where we can find general interest magazines, which reach consumers interested in a broad subject, and special interest magazines, which reach a selective segment of these consumers who are more interested in particular elements of the 
general subject ${ }^{1}$ and, therefore, who have a higher valuation of the products related to these concerns. The most interesting aspect of this relationship between degree of media specialization and consumers' valuation of quality is that it determines the way in which firms can segment the market. The following example helps us to understand this point. Consider two firms introducing a product, for example, a new computer video game or some peripheral computer equipment (e.g. a graphic accelerator card), and that firm 1 offers a low quality product while firm 2 produces a high quality good. Sellers can classify their potential consumers into those who are regular users of video games, with a high valuation of the goods, and those who are only regular users of computers, with a lower valuation. A careful selection of magazines specializing in video games (such as Computer Gaming World, PC Top Player, etc.), would allow firms to reach exclusively high-demand consumers. However, regular users of video games are also regular users of computers so, if a firm wants to reach low-demand consumers, it has to use general computer magazines (such as Computer World, Home PC, etc.), which reach both low demand and high demand consumers. As a result, the targeting technology allows firms to segment the market only by isolating the most eager customers. ${ }^{2}$

The purpose of this paper is to study the interaction between consumers and firms in this marketing environment, so we will assume that sellers can inform consumers by using either a general advertising media, which spreads the ads across the whole potential market (uniform advertising), or a more specialized media, which allows firms to concentrate their ads on a subset of consumers who value quality most (targeting). ${ }^{3}$ Taking this targeting technology as the starting point, our first goal is to solve the game in which both firms simultaneously decide their pricing and advertising (targeting) strategies for a given level of product qualities. We begin by describing the equilibrium pricing strategy when firms can use only the uniform (or general) media and both sellers compete for the marginal consumer. This solution, which is equal to the full information outcome, constitutes a reasonable benchmark against which we can compute the impact of targeting on market prices. Next, we consider that firms can either use the general media or target the ads on the subset of high valuation consumers. In this framework, the high-quality firm may have a particularly large incentive to target the advertising, given that the use of a special interest medium could improve the precision with which this seller reaches its potential customers, thus reducing the cost of informing the potential demand. The key point here is that when the high-quality firm targets the ads, the low-quality seller can use uniform advertising to reach

\footnotetext{
${ }^{1}$ For example, we can find general interest magazines devoted to sports, medicine, computers, family matters, etc., and special interest magazines devoted to specific sports (soccer, basketball, golf, etc.), medical specialities (surgery, radiology, dermatology, etc.), computer issues (videogames, Internet, etc.), or leisure activities (fitness, decoration, gardening, etc.).

${ }^{2}$ Similarly, other communication media, like cable TV or specialized radios, can allow advertisers to reach only a segment of high valuation consumers.

${ }^{3}$ An alternative interpretation of our model is to consider that all consumers are ex-ante informed of the existence of the low-quality product, for example, because in a previous stage outside the model both firms produced the same low-quality product. Then, one of the firms has to decide whether to advertise its new high-quality product to all consumers (in a wide audience medium) or to high-end consumers only (in a specialized medium).
} 
uninformed consumers who are not in the target set of the competitor, thus obtaining a captive market which arises from a new source of product differentiation: information. Accordingly, targeting divides the low-quality firm's clientele into a competitive and a captive part, so we prove that the pricing-targeted advertising game does not have a pure strategy Nash equilibrium. However, we find that, under reasonable market conditions, the game has a mixed strategy equilibrium in which the low-quality seller chooses a pure advertising strategy (uniform advertising) and randomizes between monopolizing the captive market (setting a high price) and competing with its rival for the fully informed marginal consumer (setting a low price), whereas the highquality firm randomizes between targeting the advertising campaign and launching uniform advertising, and plays a pure pricing strategy (conditional on its advertising strategy). The most interesting feature of this equilibrium with price dispersion is that the low-quality firm behaves as a pure monopoly with a positive probability, which has important implications for the pattern of price competition in the market. In particular, we find that (1) compared to the benchmark, the possibility of using targeted advertising leads both firms to always charge higher prices, (2) the increase in prices induced by targeting is more intense in highly competitive (low differentiated) markets, (3) the price of the low-quality firm is non-monotonic with the degree of product differentiation, and (4) the low-quality product may be sold at a higher expected price than the high-quality product. This means that product prices might not be correlated, or even negatively correlated, with product qualities. This conclusion is surprising, because it means that consumers allocate their financial resources inefficiently. However, there is empirical evidence of a weak and often negative relationship between price and quality, which is in line with our results. ${ }^{4}$

Our second goal is to analyze how the current proliferation of new advertising media can affect firms' ability to exercise market power. There are two ways in which this phenomenon can influence the advertising technology. First, the progressive growth of specialized advertising may well provoke a reduction in targeted advertising costs. We find that a decrease in the cost of specialized media yields higher market prices. Second, an increasing number of advertising vehicles may allow firms to control the degree of specialization of the targeting technology. The issue then is how firms choose both the optimal price and the optimal level of targeting. We show that, in order to soften price competition, the high-quality firm will choose a high or a low specialized targeting depending on whether the cost per informed consumer is non-increasing or increasing, respectively, with the level of specialization of the advertising media. In other words, the use of targeted advertising allows firms to charge higher prices and, therefore, to obtain higher profits, but firms could benefit more from using low specialized targeting.

\footnotetext{
${ }^{4}$ For example, Steenkamp (1988) analyzes the price-quality relationship in the Netherlands based on 413 product tests involving 6,580 brands and concludes that "brands exist in the marketplace that are substantially higher priced and frequently of lower quality that the cheapest-highest-quality brand available". Faulds and Lonial (2001) study the same relationship for nearly 15,400 brands of non-durable consumer products in the United States and four other developed countries-Belgium, France, the Netherlands, and Germany - and find that, for all five countries, the proportion of negative price-quality correlation exceeds $25 \%$ of the total product lines investigated.
} 
Our work is related to the literature on informative advertising and targeting. In monopolized markets, Esteban et al. (2001) build on the "constant-reach independentreadership" advertising technology formulated by Grossman and Shapiro (1984) to propose a targeting technology based on "specialized magazines with nested readerships," which allows a firm to target the ads only on those consumers with a higher valuation of the good. They show that targeting leads to a higher market price and, thus, is potentially detrimental from a welfare perspective. ${ }^{5}$ The present paper extends this analysis to a price competition framework. In a strategic context, several authors have analyzed the effects of targeting in horizontally differentiated markets. For example, Roy (2000) examines targeting in a two-stage model, where two firms first decide their advertising strategy and then compete in prices. The timing of this game suggests that advertising has a long-run nature, perhaps to create brand loyalty and, later, consumers learn prices without cost. This type of advertising allows firms to commit themselves not to invade the rival's market, so that it becomes fragmented and both firms enjoy a monopolistic position in their local markets. Iyer et al. (2005) is more related to the present paper in the sense that they also study strategic targeting when firms inform about prices and, therefore, in an environment where advertising has a short-run nature. In particular, they examine a market where each firm has a "loyal" segment of consumers and competes for another set of homogeneous consumers, who are price-sensitive. Further, each firm can target the ads on this set of low valuation consumers. Finally, Galeotti and Moraga-Gonzalez (2008) analyze short-run targeted advertising in a homogeneous good market that is segmented only with respect to the advertising media through which potential consumers can be reached by the firms.

This paper complements the literature on informative targeted advertising by studying price competition with vertically differentiated products when advertising has a short-run nature and can be targeted only on high valuation consumers. We provide new insights, which are specific to the context of vertical differentiation, about how the use of targeted advertising can affect market prices and how these prices can relate to quality levels. Further, a novel aspect of our work is that firms can choose the degree of specialization of the targeting, which allow us to investigate how sellers decide the precision of their targeted advertising campaigns. Finally, our model introduces informational differentiation into a standard model of vertical differentiation. This feature explains why the results of our work differ to a great extent from the predictions of standard vertical differentiation models.

The remainder of the paper is organized as follows. Section 2 lays the foundations of the pricing-targeted advertising model and provides some preliminary results. In Sect. 3, we first characterize a mixed strategy equilibrium where firms compete in both prices and advertising (targeting) strategies. Later, we discuss some comparative static results and analyze how firms decide the optimal level of targeting specialization. Finally, Sect. 4 closes the paper with some concluding remarks. All the proofs are relegated to an Appendix.

\footnotetext{
5 Esteban et al. (2006), adopting the same monopolistic framework, study the relationship between targeting and product quality. They find that targeted advertising has a bearing on both the price and the design of new products. Targeted advertising is also analyzed in Esteban et al. (2004) where the effects of database direct advertising are investigated.
} 


\section{The model: some preliminary results}

We consider two firms, $i=1,2$, which compete in prices for a unitary mass of consumers who demand, at most, one unit of a product. Both firms supply the same basic version of the product, except for the value of one characteristic, $q_{i}$, which measures the quality of the good, with $\Delta q=q_{2}-q_{1}>0$. For the sake of simplicity, we normalize the marginal cost of production to zero. This means, for example, that, before competition takes place, firm 2 makes a one-time fixed cost investment, which allows it to produce the high-quality product at the same cost as the low-quality product. ${ }^{6} \mathrm{~A}$ consumer's utility is $U=v+\theta q_{i}-p_{i}$ when he buys a good of quality $q_{i}$ at a price $p_{i}$, and 0 if he does not buy. The parameter $v>0$ represents consumers' common valuation of the basic product. The parameter $\theta$ of taste for quality is uniformly distributed across the population of consumers in the interval $[0,1]$, in such a way that consumers can be indexed by the value of their taste parameter. In the full information version of this model (Shaked and Sutton 1982; Tirole 1988), the consumer with a taste parameter $\theta=\frac{p_{2}-p_{1}}{\Delta q}$ is indifferent between buying the low and the high-quality good, so firm 1 and firm 2 serve the market segments $[0, \theta]$ and $[\theta, 1]$, respectively.

Consumers are unaware of the existence, the quality and the price of the goods, so a potential consumer cannot buy the product unless sellers invest in advertising. ${ }^{7}$ In order to inform consumers, the firms can use either the uniform media, which spread the ads to the entire population of potential buyers $[0,1]$, or the specialized media, which reach the consumers in the interval $[z, 1]$, where $1>z>0$ measures the level of specialization of the targeting. We will represent the set of pure advertising/targetingstrategies as $t_{i}=\{0, z\}$, where 0 denotes the decision to use uniform advertising and $z$ denotes the decision to target the campaign. ${ }^{8}$ We begin by considering that firms have only one specialized medium available, and that the value of $z$ is exogenous. Later, we assume that firms have an array of targeting possibilities available with different levels of specialization, $z \in\left\{z_{0}, z_{1}, z_{2}, \ldots, z_{n}\right\}$, so they can choose the optimal degree of specialization of the advertising campaign, $z=z^{*}$. Finally, we consider that, when a firm advertises the product in a segment of the market, all consumers in that segment become informed about the existence, price and characteristics of the good. ${ }^{9}$ Advertising is costly and the cost of a campaign depends on the size of the target market.

\footnotetext{
${ }^{6}$ The model and the results can be easily extended to the case that producing higher quality is more expensive. However, this extension does not provide any new interesting insight.

7 This means that consumers' search cost is high relative to the expected surplus offered by the goods so, in the absence of information, consumers do not purchase any good (see, for example, Grossman and Shapiro 1984; Stegeman 1991; Stahl 1994).

8 We note first that the simplifying assumption of a binary targeting choice is not restrictive. Esteban et al. (2006) show that this type of analysis can be extended to the case in which a firm simultaneously uses multiple advertising media with different target audiences. Second, we note that the use of both mass and specialized advertising only makes sense if the firm price discriminates (we analyze this possibility in a separate paper, Esteban and Hernández 2007). In the present work, we study targeting under uniform pricing, which is the mainstream case of most product markets where the good is sold to consumers through traditional retail channels.

${ }^{9}$ With this simplifying assumption, we discard the analysis of optimal advertising intensities in order to focus the model on the study of optimal pricing strategies (see Iyer et al. 2005; Galeotti and Moraga-Gonzalez 2008).
} 
If $A_{0}$ denotes the cost of a uniform advertising campaign and $A_{1}=A_{1}(z)$ denotes the cost of a campaign targeted on $[z, 1]$, then, given that targeted advertising reduces the number of consumers reached by the campaign, we assume ${ }^{10}$ that $A_{1}^{\prime}(z)<0$, $\Delta A=A_{0}-A_{1}>0$ for all $1>z>0$, and $\operatorname{Lim}_{z \longrightarrow 0} A_{1}(z)=A_{0}$.

Having specified the fundamentals of the model, we now analyze the simultaneous move game ${ }^{11}$ in which both firms decide their pricing-targeted advertising strategies, $s_{i}=\left(p_{i}, t_{i}\right)$. For future reference, let $\left(p_{1}^{m}, p_{2}^{m}\right)$ denote the unique equilibrium price strategies when both firms can use only uniform advertising and compete for the fully informed marginal consumer, $\theta^{m}=\frac{p_{2}^{m}-p_{1}^{m}}{\Delta q}$ (see the Appendix for details of this equilibrium). We assume that $\Pi_{1}^{m}=\frac{\Delta q-9 A_{0}}{9} \geq 0$ and $v>\frac{\Delta q}{3}$, so that a full information equilibrium, where firm 1 and firm 2 achieve non-negative profits and serve the market segments $\left[0, \theta^{m}\right]$ and $\left[\theta^{m}, 1\right]$, respectively, exists. Taking the uniform advertising equilibrium as the reference point, let us assume that there is a specialized advertising medium which allows firms to target the ads on the subset of high valuation consumers $[z, 1]$. In order to understand how targeting affects the pattern of price competition in the market, it is instructive to begin by considering that firms can target the ads on the market segment $\left[\theta^{m}, 1\right]$. In this case, we first prove that the game does not have an equilibrium in which both firms use uniform advertising.

Lemma 1 If $z \longrightarrow \theta^{m}$, a pure strategy Nash equilibrium with $\left(t_{1}=0, t_{2}=0\right)$ does not exist.

Lemma 1 simply suggests that there is a high incentive to target the advertising in order to improve advertising cost efficiency. More precisely, targeting is particularly attractive for firm 2 (the high-quality firm), given that it allows this seller to save advertising costs by focusing the ads on its potential consumers; by contrast, firm 1 must use uniform advertising to reach its low-valuation potential demand. In fact, the proof of Lemma 1 shows that, given $s_{1}=\left(p_{1}^{m}, t_{1}=0\right)$, firm 2's best response is $s_{2}=\left(p_{2}^{m}, t_{2}=z\right)$ and, therefore, it makes sense to look for an equilibrium strategy profile with $\left(t_{1}=0, t_{2}=z\right)$. Nevertheless, the following Lemma states that if $t_{2}=z$, it is not possible to sustain a market equilibrium in which $t_{1}=0$.

Lemma 2 If $z \longrightarrow \theta^{m}$, a pure strategy Nash equilibrium with $\left(t_{1}=0, t_{2}=z\right)$ does not exist.

The intuition of this Lemma is as follows. Starting from the benchmark, where firms can use only uniform advertising, when sellers can target their ads on $z \longrightarrow \theta^{m}$, and given $s_{1}=\left(p_{1}^{m}, t_{1}=0\right)$, firm 2's best response is $s_{2}=\left(p_{2}^{m}, t_{2}=z\right)$, which causes products to be differentiated along two dimensions, quality and information, thus substantially changing the pattern of price competition in the market. The important point

$\overline{10}$ Esteban et al. $(2001,2006)$ provide empirical evidence confirming this intuition for the case "specialized magazines with nested readerships".

11 Given that the ads inform about prices, it seems natural to consider that firms set prices without observing the competitor's advertising strategy. This approach is usually followed in the literature on targeted advertising (see, for example, Grossman and Shapiro 1984; Iyer et al. 2005; Galeotti and Moraga-Gonzalez 2008). 
is that, given this $s_{2}$, firm 1's best response is to play $s_{1}=\left(p_{1}^{M}, t_{1}=0\right)$, that is to say, the use of targeted advertising naturally leads firm 1 to charge the monopoly price to the segment of imperfectly informed consumers, $[0, z]$ and, therefore, we should look for an equilibrium in which the firm monopolizes this captive market. The problem with this outcome is that both products are strategic complements so, if $t_{2}=z$, firm 2 would respond to the monopolization of $[0, z]$ by raising the price, $p_{2}>p_{2}^{m}$. This will lead firm 1 to compete for the segment of the market $[z, 1]$ by lowering the price $p_{1}$ $<p_{1}^{M}$ which, in turn, will induce firm 2 to also lower the price. Finally, firm 1's best response to this latter strategy would be, once more, to monopolize $[0, z]$ by raising the price to $p_{1}^{M}$, thus starting the same price competition-cycle again. This implies that the existence of an equilibrium where firms play $\left(t_{1}=0, t_{2}=z\right)$ and firm 1 can monopolize the captive market must be based on mixed strategies.

\section{Equilibrium in mixed strategies}

Before solving the game, we first note that, given $t_{2}=z$, for sufficiently low values of $z$ (for example, when $z \longrightarrow 0$ ), firm 1 will not find it optimal to monopolize the captive market, $[0, z]$, so both firms will compete for the indifferent consumer, $\theta=\frac{p_{2}-p_{1}}{\Delta q}$, by charging the full information prices, $\left(p_{1}^{m}, p_{2}^{m}\right)$. This means that an equilibrium where firm 1 monopolizes the captive market can exist only if $z$ is not very low, $z>\underline{z}$. To find this equilibrium, we begin by noting that, according to the marketing literature, many firms use a mix of advertising media to achieve maximum consumer reach and, at the same time, minimum advertising cost (Kerin et al. 2009; Kotler and Armstrong 2007). In our model, firm 1 can reach its potential customers, $[0, \theta]$, only by using uniform advertising, which reaches the whole potential market, so it is clear that this firm will play a pure advertising strategy, $t_{1}=0$. By contrast, firm 2 can reach its "natural" market $[\theta, 1]$ by using either uniform or targeted advertising, so it makes sense to think that this seller mixes both types of media. Therefore, we must look for a hybrid equilibrium in which firm 1 plays a pure advertising strategy, $t_{1}=0$, and firm 2 a mixed advertising strategy. More precisely, if $z$ is not very high, firm 2 has, a priori, a clear incentive to set $t_{2}=z$ in order to reach its customers with the minimum advertising cost. Under these pure advertising strategies, $\left(t_{1}=0, t_{2}=z\right)$ firm 1 has a captive market, $[0, z]$, which it can monopolize so, if $z>\underline{z}$, firm 1 will set $p_{1}=p_{1}^{M}$. The key point is that, in response to this monopolization strategy, firm 2 might well find it optimal to set $t_{2}=0$, incurring a higher advertising cost, in order to attract the consumers in $[0, z]$. Taking this into account, it is reasonable to think that firm 1 will prevent firm 2 from predicting its pricing behavior by playing a mixed pricing strategy, with $p_{1} \leq p_{1}^{M}$, and that, in response to this, firm 2 will play $t_{2}=z$ with a positive probability, so as to reduce advertising expenses. Based on this reasoning, the equilibrium must involve firm 2 randomizing in the advertising strategy (between using uniform and targeted advertising) and firm 1 in the pricing strategy (between monopolizing the captive market, $[0, z]$, and competing for the segment $[z, 1])$.

In order to characterize this equilibrium, let us denote by $p_{2}(z)$ and $p_{2}(0)$ the prices charged by firm 2 when $t_{2}=z$ and $t_{2}=0$, respectively, and by $\theta(z)=\frac{p_{2}(z)-p_{1}}{\Delta q}$ 
the marginal buyer when $t_{2}=z$ and both firms compete for the market segment $[z, 1]$. Under the condition $z \leq \theta(z)$, when $t_{2}=z$ and both firm compete in prices, the resulting profit maximization problem yields a marginal consumer, which we denote by $\theta^{0}(z)$. If the condition $z \leq \theta^{0}(z)$ indeed holds, then, in equilibrium, firm 2's advertising campaign reaches all the consumers who are willing to purchase its product, $\left[\theta^{0}(z), 1\right]$. However, if the specialization of the targeting is high, i.e. $z>\theta^{0}(z)$, when $t_{2}=z$, firm 2 can reach only a fraction of the potential demand $\left[\theta^{0}(z), 1\right]$, so the pattern of price competition changes thus yielding a different equilibrium marginal consumer, $\theta^{1}(z) \neq \theta^{0}(z)$. This means that the pattern of competition between firms will be different depending on whether $z \leq \theta^{0}(z)$ or $z>\theta^{0}(z)$. Therefore, we next characterize the two types of equilibria separately.

Proposition 1 If $\underline{z}<z \leq \theta^{0}(z)=\frac{2}{6-\alpha^{0}\left(3+\beta^{0}\right)}$, there is a non-empty set of parameters $\left[v, q_{1}, \Delta q, A_{0}, \Delta A\right]$ for which the following mixed strategy Nash equilibrium exists:

(i) Firm 1 only uses uniform advertising $\left(t_{1}=0\right)$ and it charges $p_{1}^{M}=v$ with probability $\alpha^{0} \in(0,1)$ and $p_{1}^{0}=\frac{\Delta q\left(1-\alpha^{0} \beta^{0} z\right)}{3\left(1-\alpha^{0}\right)}$ with probability $\left(1-\alpha^{0}\right)$.

(ii) Firm 2 chooses $t_{2}=z$ and $p_{2}^{0}(z)=\frac{\Delta q\left[4-\alpha^{0} \beta^{0} z-3 \alpha^{0} z\right]}{6\left(1-\alpha^{0}\right)}$, with probability $\beta^{0} \in(0,1)$, and $t_{2}=0$ and $p_{2}^{0}(0)=\frac{\Delta q\left(4-\alpha^{0} \beta^{0} z\right)}{6\left(1-\alpha^{0}\right)}$ with probability $\left(1-\beta^{0}\right)$.

Finally, $\beta^{0}\left(\alpha^{0}\right)=\frac{\alpha^{0} \Delta q z\left(8-3 \alpha^{0} z\right)-12 \Delta A\left(1-\alpha^{0}\right)}{2 \Delta q\left(\alpha^{0}\right)^{2} z^{2}}$ and $\alpha^{0}$ is implicitly defined by:

$$
9 \beta^{0}(\alpha) v z(1-\alpha)^{2}-\Delta q\left(1-\alpha \beta^{0}(\alpha) z\right)^{2}=0
$$

Proposition 1 states that, when $\theta^{0}(z) \geq z>\underline{z}$, the game has an equilibrium with price dispersion in which firm 1's mixed pricing strategy entails a two-point distribution involving a randomization between monopolizing the captive market $[0, z]$ by setting a high price, $p_{1}=p_{1}^{M}$, with probability $\alpha^{0}$, and competing for the segment of the market $[z, 1]$ by setting a low price, $p_{1}=p_{1}^{0}$, with probability $\left(1-\alpha^{0}\right)$. This means that firm 1 follows a high-low pricing strategy: it charges the monopoly price to squeeze more surplus from its captive customers but, to prevent firm 2 from poaching its customers, it also quotes a lower price for the same product. On the other hand, firm 2 faces a trade-off between using targeted advertising, in order to reduce advertising expenses, and uniform advertising, in order to attract customers in $[0, z]$ who are ready to buy firm 1's product at the monopoly price. As a result, the firm randomizes between sending uniform and targeted advertising and always competes for the segment of consumers $[z, 1]$ by playing a pure pricing strategy, conditional on the advertising strategy, $t_{2}=z$ or $t_{2}=0$.

Next, we characterize the equilibrium when $z>\theta^{0}(z)$. Before studying this scenario, we first note that, if $z$ is sufficiently high (for example, when $z \longrightarrow 1$ ), firm 2 will never find it optimal to target the advertising campaign, so both firms will set $t_{1}=t_{2}=0$ and charge the full information prices, $\left(p_{1}^{m}, p_{2}^{m}\right)$. This means that an equilibrium with $z>\theta^{0}(z)$ and firm 2 targeting the advertising can exist only if $z$ is not very high, $z \leq \bar{z}$. The following proposition describes this equilibrium. 
Proposition 2 If $\bar{z} \geq z>\theta^{0}(z)=\frac{2}{6-\alpha^{0}\left(3+\beta^{0}\right)}$, there is a non-empty set of parameters $\left[v, q_{1}, \Delta q, A_{0}, \Delta A\right]$ for which the following mixed strategy Nash equilibrium exists:

(i) Firm 1 only uses uniform advertising $\left(t_{1}=0\right)$ and it charges $p_{1}^{M}=v$ with probability $\alpha^{1} \in(0,1)$ and $p_{1}^{1}=\frac{\Delta q\left[1-\beta^{1}+2 \beta^{1} z\left(1-\alpha^{1}\right)\right]}{\left(1-\alpha^{1}\right)\left(3-\beta^{1}\right)}$ with probability $\left(1-\alpha^{1}\right)$.

(ii) Firm 2 chooses $t_{2}=z$ and $p_{2}^{1}(z)=p_{1}^{1}+z \Delta q$, with probability $\beta^{1} \in(0,1)$, and $t_{2}=0$ and $p_{2}^{1}(0)=\frac{\Delta q\left[2-\beta^{1}+\beta^{1} z\left(1-\alpha^{1}\right)\right]}{\left(1-\alpha^{1}\right)\left(3-\beta^{1}\right)}$ with probability $\left(1-\beta^{1}\right)$.

Finally, $\left(\alpha^{1}, \beta^{1}\right)$ are defined by:

$$
\begin{aligned}
& v z \beta(1-\alpha)^{2}(3-\beta)^{2}-\Delta q[1-\beta+2 \beta z(1-\alpha)]^{2}=0, \\
& \Delta q-\Delta A(1-\alpha)(3-\beta)^{2}-\Delta q z(6-\alpha(9-\beta(4-\beta))) \\
& \quad+\Delta q z^{2}(1-\alpha)\left(9-\alpha \beta^{2}\right)=0
\end{aligned}
$$

Proposition 2 states that, if $z>\theta^{0}(z)$, which requires that $z>\frac{1}{3}$, there is also an equilibrium in which firm 1 follows a high-low pricing strategy and firm 2 randomizes between sending uniform and targeted advertising. However, compared to the case that $z \leq \theta^{0}(z)$, the pattern of price competition between firms changes. Under the condition $z>\theta^{0}(z)$, when firm 2 sets $t_{2}=z$ and competes for the segment of consumers $\left[\theta^{0}(z), 1\right]$, the advertising campaign cannot reach the complete set of consumers who are willing to buy firm 2's product. This generates a first-order effect on the price, which induces firm 2 to raise $p_{2}(z)$ up to the level where the marginal buyer, $z$, is indifferent between the two firms' products, that is, the equilibrium marginal buyer ${ }^{12}$ is now $\theta^{1}(z)=\frac{p_{2}^{1}(z)-p_{1}^{1}}{\Delta q}=z$. This implies that, for a given $(\alpha, \beta)$, compared to the case where $z \leq \theta^{0}(z)$, firm 1 faces less price competition and, given that the products are strategic complements, an increase in $p_{1}$ yields a higher $p_{2}(0)$. Therefore, we conclude that the switch from $z \leq \theta^{0}(z)$ to $z>\theta^{0}(z)$ gives firms an incentive to raise prices.

In summary, from Propositions 1 and 2 it follows that, regardless of whether $z \lessgtr$ $\theta^{0}(z)$, in equilibrium, the high-quality firm randomizes in the advertising strategy and both firms compete by implementing a high-low pricing strategy. This pattern of competition seems quite realistic. ${ }^{13}$ Firm 2's strategy is a good reflection of the dilemma between increasing consumer reach to a maximum (by lowering prices and/or expanding the advertising coverage) and decreasing advertising costs to a minimum (by targeting the advertising), a trade-off which, according to the marketing literature, is central in the media planning problem. Further, the more specialized medium

\footnotetext{
12 Note that, considering Propositions 1 and 2 together, the equilibrium marginal consumer when $t_{2}=z$ is $\theta^{*}(z)=\operatorname{Max}\left[\theta^{0}(z), z\right]$.

13 We note that the equilibrium results can be extended to a market with more than two competitors, as long as the targeting technology is nested. In this scenario, the higher-quality firms will exploit the higher cost efficiency of targeting by focusing their advertising campaigns on their set of selective customers, thus giving the lowest end of the market to the lowest-quality firm. This firm will monopolize its captive market with a positive probability, which makes the lowest-quality firm a soft competitor, so the remaining results (prices rise, firms play mixed strategy, etc.) should readily follow.
} 
concentrates on the advertising of high-quality products whereas the less specialized medium also advertises basic goods, which seems consistent with the empirical evidence.

Regarding the existence of the equilibria, the analytical complexity of (1), (2) and (3) prevents us from obtaining a tractable explicit solution for $(\alpha, \beta)$, so it is not possible to provide general existence conditions. Therefore, we must base the existence analysis on the calibration of the model. In order to set a meaningful value for the parameters, we base the simulation exercise on a real-world example applied to our model. We consider two firms producing a computer graphic accelerator card (GAC), which differs in the value of one characteristic: the video memory (VRAM). The memory capacity of most modern video cards ranges from $512 \mathrm{Mb}$ to $2 \mathrm{~Gb}$, so we set $q_{1}=512 \mathrm{Mb}$ and a maximum value of $q_{2}=\bar{q}_{2}=2 \mathrm{~Gb} \approx 4 q_{1}$. Our first goal is to analyze the robustness of the mixed strategy equilibria to changes in the differentiation parameter within the range $\Delta q \in\left(0, \bar{q}_{2}-q_{1}\right]$. Considering that the value of the quality taste parameter is normalized to $\theta \in[0,1]$, to obtain the monetary valuation of quality, $\theta q_{i}$, we need to rescale the variable $q_{i}$. To that end, it seems logical to think that the maximum valuation of the quality attribute should not exceed the valuation of the basic version of the product. Accordingly, we assume that, for any $\theta \in[0,1]$, it holds that $\theta q_{i} \leq v$, so we fix $\bar{q}_{2}=v$. Regarding the value of $v$, we note that, in the Spanish computer market, the average price of a GAC ranging between $512 \mathrm{Mb}$ and $2 \mathrm{~Gb}$ is around $\bar{p}=225 €$, so it seems reasonable to set $v=2 \bar{p}=550 €$. Therefore, we have that $v=550, q_{2} \leq \bar{q}_{2}=550$ and $q_{1}=\frac{\bar{q}_{2}}{4}=137.5$, which means that $\Delta q \in(0,412.5]$. However, the condition $\Pi_{1}^{m} \geq 0$ determines a new lower bound for $\Delta q, \Delta q \geq 9 A_{0}$, i.e. $\Delta q \in\left[9 A_{0}, \bar{q}_{2}-q_{1}\right]$. In order to set a reasonable value for $A_{0}$, we follow the marketing literature, which reports that a firm launching a new product usually incurs an advertising cost that represents an average of about $20 \%$ of sales (Kotler and Armstrong 1998). Taking into account that, for a given $\Delta q$, the average sales in the industry when firms use mass advertising are $\frac{5 \Delta q}{18}$, we run the simulation considering the average quality differential:

$$
A_{0}=0.2 \frac{5\left[\frac{9 A_{0}+\left(\bar{q}_{2}-q_{1}\right)}{2}\right]}{18} .
$$

From this equation, we obtain $A_{0}=\frac{\bar{q}_{2}-q_{1}}{27}$, so a reasonable range of values of $\Delta q$ is $\Delta q \in\left[9 A_{0}, \bar{q}_{2}-q_{1}\right]=[137.5,412.5]$. Regarding the value of $A_{1}$, we assume that the targeted advertising cost is linearly related to the size of the market, i.e. $A_{1}=A_{0}(1-z)$. Considering these parameter values, we first compute the model when $z \leq \theta^{0}(z)$, which implies that $z \leq \frac{2}{6-\alpha^{0}\left(3+\beta^{0}\right)}>\frac{1}{3}$, so this condition holds for any $z \leq \frac{1}{3}$. Accordingly, we perform the simulation for a lower $z, z=\frac{1}{4}$. To sum up, we begin by analyzing the existence of the equilibrium described in Proposition 1 for the following base-case market scenario, $\left[v, q_{1}, \bar{q}_{2}\right]=[550,137.5,550]$, and for $z=0.25, A_{0}=\frac{\bar{q}_{2}-q_{1}}{27}, A_{1}=A_{0}(1-z)$ and, finally, for $\Delta q \in[137.5,412.5]$. Under 
these market conditions, we find that this equilibrium exists ${ }^{14}$ for $\Delta q \in[146.6,412.5]$. When $z>\theta^{0}(z)$, the simulation of the model, for a higher value of $z, z=0.45$, and the same market scenario, yields that the equilibrium described in Proposition 2 exists for all $\Delta q \in\left[9 A_{0}, \bar{q}_{2}-q_{1}\right]=[137.5,412.5]$. All this suggests that the solution to the game exists for a wide set of reasonable market conditions. ${ }^{15}$

The second goal of our simulation exercise is to determine the range of values of $z$ for which each of the two equilibria exists. The computation of our model for the same base-case market scenario, but considering an intermediate value of the quality difference, $\Delta q=300$, and $A_{0}=0.2 \frac{5 \Delta q}{18}=\frac{\Delta q}{18}, A_{1}=A_{0}(1-z)$, yields that the first equilibrium exists for all $0.07<z \leq 0.347$, so Proposition 1 describes the equilibrium for reasonable targeting technologies, $z>0.07$, when the targeted market is not small, $1-z \geq 0.653$. Further, under the same market scenario, we find that the equilibrium described in Proposition 2 exists for all $0.347<z \leq \bar{z}=0.49$, whilst, for higher levels of $z$, the model generates the full information outcome.

Having described the solution of the game, we next discuss the impact of targeting on market prices. The first interesting result is that, in both equilibria, there is a positive probability that the low-quality firm behaves as a pure monopoly. This means that the transition from uniform advertising to targeted advertising turns a pure vertically differentiated market into a hybrid market incorporating some features of a monopoly, which has important implications for the pattern of price competition in the market. The following proposition addresses this point.

Proposition 3 The equilibrium prices satisfy: $p_{1}^{M}>p_{1}^{k}>p_{1}^{m} ; p_{2}^{k}(0)>p_{2}^{m}$, $p_{2}^{k}(z)>p_{2}^{m}$, for $k=0,1$, and $p_{2}^{0}(0)>p_{2}^{0}(z)$.

Proposition 3 proves that, compared to the case where firms can use only uniform advertising, the possibility of using targeting leads both firms to always charge higher prices. Indeed, both firms raise their prices even when they use uniform advertising, which occurs with probability $(1-\beta)$. To understand the intuition behind these results we note that, when firm 1 plays $p_{1}<p_{1}^{M}$, the pattern of price competition with firm 2 is the same as when deciding $p_{1}^{m}$ in the sense that, in both cases, firm 1's reaction function is identical (see (32) and (33) in the Appendix) and, therefore, the firm faces the "standard" competition scenario of uniform advertising, with a demand:

$$
D_{1}=\beta\left(\frac{p_{2}(z)-p_{1}}{\Delta q}\right)+(1-\beta)\left(\frac{p_{2}(0)-p_{1}}{\Delta q}\right)=\left(\frac{E p_{2}-p_{1}}{\Delta q}\right) \text {. }
$$

By contrast, firm 2 faces a mixed competitive scenario. When firm 1 sets the monopoly price, firm 2 captures the entire informed market so, with probability $\alpha$, firm 2 faces a

\footnotetext{
14 For a small set of low values of $\Delta q, \Delta q \in[137.5,146.6)$, the equilibrium values of $\alpha$ turn out to be relatively high so, when $t_{2}=0$, firm 2 finds it optimal to deviate by setting the monopoly price, $p=v$. However, if we consider different market conditions, for example, if the advertising cost represents $25 \%$ of the average sales or if the targeted advertising cost function is $A_{1}=A_{0}(1-z)^{0.4}$, we find that the equilibrium exists for all $\Delta q \in\left[9 A_{0}, \bar{q}_{2}-q_{1}\right]$.

15 Indeed, extensive simulations of our model confirm that the equilibrium is quite robust to changes in all the parameter values.
} 
totally inelastic demand. At the same time, with probability $(1-\alpha)$, firm 2 faces competition, i.e. $p_{1}<p_{1}^{M}$. In this case, when $z \leq \theta^{0}(z)$, in equilibrium, the high-quality firm always finds it optimal to compete for the fully informed marginal consumer, $\left(\frac{p_{2}-p_{1}^{0}}{\Delta q}\right)$, regardless of the type of advertising used, so it faces a more elastic demand which also corresponds to the "standard" competition scenario of uniform advertising. This means that, when deciding the optimal prices $\left(p_{1}^{0}, p_{2}^{0}(0), p_{2}^{0}(z)\right)$, firm 1 faces the same demand as with uniform advertising whilst firm 2 faces the following overall more inelastic demand:

$$
D_{2}= \begin{cases}{\left[\alpha(1-z)+(1-\alpha)\left(1-\frac{p_{2}(z)-p_{1}^{0}}{\Delta q}\right)\right],} & \text { if } t_{2}=z \\ {\left[\alpha+(1-\alpha)\left(1-\frac{p_{2}(0)-p_{1}^{0}}{\Delta q}\right)\right],} & \text { if } t_{2}=0\end{cases}
$$

thus explaining $p_{2}^{0}(0)>p_{2}^{m}$ and $p_{2}^{0}(z)>p_{2}^{m}$. Further, firm 2's demand when $t_{2}=0$ is higher than when $t_{2}=z$, which explains $p_{2}^{0}(0)>p_{2}^{0}(z)$. Finally, given that the two goods in the market are strategic complements, if firm 2 charges higher prices with targeting, firm 1 will react by raising its price, i.e. $p_{1}^{0}>p_{1}^{m}$. When $z>\theta^{0}(z)$, the emergence of a new first-order effect of targeting on $p_{2}(z)$ gives firms an extra incentive to raise prices, so we also find that $p_{2}^{1}(0)>p_{2}^{m}, p_{2}^{1}(z)>p_{2}^{m}$ and $p_{1}^{1}>p_{1}^{m}$. However, due to the first-order effect on prices, the simulation of our model yields that $p_{2}^{1}(0) \gtrless p_{2}^{1}(z)$.

It is instructive to compare these results with the related literature. In Iyer et al. (2005) and Galeotti and Moraga-Gonzalez (2008), firms play a symmetric pricingadvertising game which yields an equilibrium with atomless mixed price distributions. By contrast, our model of pricing-advertising competition is, by nature, asymmetric, and this asymmetry generates a price distribution with a mass point at the monopoly level (see Narasimhan 1988), which substantially softens the intensity of price competition in the market. Further, we show that targeting can generate a first-order effect on prices, which provides an extra incentive to raise prices. This means that some marketing environments (vertically differentiated markets, nested advertising media and highly specialized targeting) are especially appropriate for firms to substantially increase their ability to exercise market power. Starting from this point, it is interesting to determine how the firms' optimal prices relate to the market conditions. Next, we carry out a comparative static exercise to study this issue in detail.

\subsection{Comparative static results}

In this section, we study the relationship between the optimal prices and (1) the quality levels, (2) the degree of product differentiation and (3) the cost-efficiency of the targeting technology. Given the complexity of (1), (2) and (3), we cannot obtain a tractable analytical explicit solution for $\alpha, \beta$ so, in what follows, we revert to a calibration of the model. Obviously, this type of analysis takes away generality from our work but, 


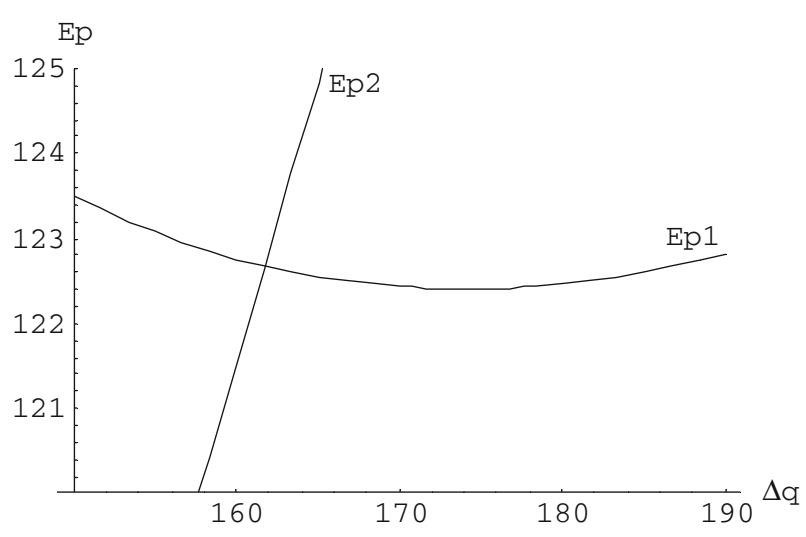

Fig. 1 Comparative statics $(\Delta q, E p)$

in exchange, it does yield some interesting insights about how targeting can affect the market outcome.

Regarding the relationship between prices and product quality, the literature on vertical differentiation provides two standard results, (1) that the high-quality firm always charges a higher price, and (2) that prices are monotonic in the degree of product differentiation. Our work reveals that the introduction of informational differentiation into a standard vertical differentiation model can substantially change these results. Figure 1 illustrates the optimal expected prices for the base-case market scenario, $\left[v, q_{1}, \bar{q}_{2}\right]=[550,137.5,550]$, with $A_{0}=\frac{\bar{q}_{2}-q_{1}}{27}, A_{1}=A_{0}(1-z)$, $z=0.25<\theta^{0}(z)$ and, when market competition is strong, that is to say, when the degree of product differentiation is relatively low, $190 \geq \Delta q \geq 146.6$.

Two important messages can be obtained with the help of this figure. First, the figure reflects well that the relationship between the optimal expected price of the low-quality firm and the parameter $\Delta q$ is non-monotonic. This result is surprising and, therefore, it is important to identify the different forces at work which justify this non-monotonic behaviour. Taking into account that $E p_{1}=\alpha(\Delta q) p_{1}^{M}+[1-\alpha(\Delta q)]$ $p_{1}^{0}[\alpha(\Delta q), \beta(\Delta q), \Delta q]$, we have that:

$$
\frac{\partial E p_{1}}{\partial \Delta q}=\frac{\partial \alpha}{\partial \Delta q}\left[p_{1}^{M}-p_{1}^{0}\right]+(1-\alpha) \frac{\partial p_{1}^{0}}{\partial \alpha} \frac{\partial \alpha}{\partial \Delta q}+(1-\alpha) \frac{\partial p_{1}^{0}}{\partial \beta} \frac{\partial \beta}{\partial \Delta q}+(1-\alpha) \frac{\partial p_{1}^{0}}{\partial \Delta q} .
$$

We note that, as $\Delta q$ increases, price competition is softened, so firm 1 progressively finds competitive pricing more profitable than monopoly pricing. This means that we can expect firm 2 to have a lower incentive to compete for $[0, z]$, i.e. $\beta$ increases. At the same time, given that, compared to the case where $t_{2}=z$, when $t_{2}=0$ firm 2 competes with firm 1 for a greater demand, a higher $\Delta q$ increases $\Pi_{2}(0)$ more than $\Pi_{2}(z)$, where $\Pi_{2}(0)$ and $\Pi_{2}(z)$ represent firm 2's profits when $t_{2}=0$ and $t_{2}=z$, respectively. In this scenario, we can expect that firm 1 will have a lower incentive to monopolize the 
captive market, so $\alpha$ falls. Given these results ${ }^{16}\left(\frac{\partial \alpha}{\partial \Delta q}<0\right.$ and $\left.\frac{\partial \beta}{\partial \Delta q}>0\right)$, and that $\frac{\partial p_{1}^{0}}{\partial \alpha}>0$ and $\frac{\partial p_{1}^{0}}{\partial \beta}<0,(6)$ indicates that there is an interplay between a positive direct effect of $\Delta q$ on the price, $\frac{\partial p_{1}^{0}}{\partial \Delta q}>0$, and three negative indirect effects of $\Delta q$ on $E p_{1}$ through $\alpha$ and $\beta$. We now discuss the nature of these effects: (1) given the probabilities $\alpha$ and $\beta$, the last term in (6) corresponds to the typical direct effect of an increase in the degree of product differentiation reducing the extent of price competition in the market, thus leading firm 1 to charge a higher $p_{1}^{0}$ and, hence, a higher $E p_{1}$, (2) the lower $\alpha$ along with the higher $\beta$, induced by a higher $\Delta q$, decrease the probability that firm 1 charges the monopoly price and increase the elasticity of the demands faced by the firms when competing for the marginal consumer. These indirect effects tend to lower $E p_{1}$. When $\Delta q$ is sufficiently low, $174 \geq \Delta q \geq 146.6$, the sum of the indirect effects is dominant, so a higher degree of product differentiation leads firm 1 to set a lower expected price. However, as $\Delta q$ increases, the indirect effects lose strength so the direct effect dominates and we obtain the standard result that a higher $\Delta q$ yields higher prices.

The second message obtained from Fig. 1 is that, for a sufficiently low $\Delta q, 161 \geq$ $\Delta q \geq 146.6$, the low-quality firm sets (in expected terms) a higher price than the high-quality firm, $E p_{1}>E p_{2}$. The intuition behind this result is as follows. Whilst, in equilibrium, the high-quality firm always competes for the fully informed marginal consumer, firm 1 mixes competitive pricing and pure monopoly pricing. When the degree of product differentiation is low, competitive pricing, i.e. $\left(p_{1}^{0}, p_{2}^{0}(0), p_{2}^{0}(z)\right)$, yields low profits, and the weight of the monopolistic behaviour turns out to be relatively high ( $\alpha$ is relatively high). In this case, the low-quality firm sets a substantially high price which might well exceed the price of the high-quality firm. However, as $\Delta q$ increases, the competitive behaviour increasingly gains weight and the model approaches a standard vertical differentiation model with the typical result that prices increase in $\Delta q$ and $E p_{2}>E p_{1}$. Accordingly, we find that, compared to uniform advertising, the increase in prices induced by targeting will be more intense in highly competitive markets where $\Delta q$ is low. In other words, targeting turns out to be an effective mechanism to soften price competition in markets with a low differentiation. ${ }^{17}$ Finally, we note that all these comparative static results also apply to the case where $z>\theta^{0}(z)$. For example, when $z=0.45$ and firms compete in the same market scenario, we find that, if $146>\Delta q>137.5$, then $E p_{1}>E p_{2}$, and $E p_{1}$ reaches a minimum at $\Delta q=191$.

Next, we study how market prices relate to advertising cost efficiency. It is clear that the current increasing number of specialized media is likely to progressively reduce the cost of a specialized advertising campaign, so an interesting question to analyze is how a higher advertising cost efficiency affects both firms and consumers. Table 1 addresses this issue by showing how both expected prices and $(\alpha, \beta)$ vary with $A_{1}$

\footnotetext{
16 Extensive simulations of the model confirm that $\frac{\partial \alpha}{\partial \Delta q}<0$ and $\frac{\partial \beta}{\partial \Delta q}>0$.

17 For example, if $\Delta q=147$, the switch from uniform to targeted advertising increases $E p_{1}$ and $E p_{2}$ around 153 and $15 \%$, respectively, whereas, if $\Delta q=190$, these percentages are reduced to 94 and $12 \%$.
} 
Table 1 Targeting costs and prices

\begin{tabular}{lrllll}
\hline$z$ & $A_{1}$ & $\alpha$ & $\beta$ & $E p_{1}$ & $E p_{2}$ \\
\hline 0.25 & 12 & 0.086 & 0.286 & 146.75 & 217.49 \\
0.25 & 10 & 0.119 & 0.307 & 164.57 & 224.95 \\
0.25 & 8 & 0.150 & 0.327 & 181.22 & 232.38 \\
0.25 & 6 & 0.179 & 0.348 & 196.82 & 239.77 \\
0.45 & 12 & 0.002 & 0.144 & 104.65 & 207.47 \\
0.45 & 10 & 0.026 & 0.151 & 118.18 & 212.42 \\
0.45 & 8 & 0.050 & 0.159 & 131.06 & 217.33 \\
0.45 & 6 & 0.073 & 0.166 & 143.31 & 222.19 \\
\hline
\end{tabular}

for $\left[v, q_{1}, \bar{q}_{2}\right]=[550,137.5,550]$, an intermediate level of product differentiation, $\Delta q=300, A_{0}=\frac{\Delta q}{18}$, and $z=0.25<\theta^{0}(z), z=0.45>\theta^{0}(z)$.

We find that, when the cost of specialized advertising drops, market prices increase. This occurs because cheaper targeting directly increases $\Pi_{2}(z)$, so firm 2 finds $t_{2}=z$ more attractive than $t_{2}=0$. As a result, we can expect firm 1 to have a higher incentive to monopolize its captive market, i.e. $\alpha$ rises, in such a way that firm 2 faces more inelastic demands. As a result, firm 2 raises $p_{2}(0)$ and $p_{2}(z)$, which, given that both products are strategic complements, leads firm 1 to raise $p_{1}$. Therefore, a higher advertising cost efficiency improves the firms' ability to exercise market power which, of course, decreases consumers' surplus.

\subsection{Optimal targeting}

The huge current proliferation of new information technologies implies that, to reach their potential customers, firms may well find a set of different specialized advertising outlets available and, therefore, another interesting issue to study is how firms choose the optimal degree of targeting specialization, $z=z^{*}$. We endogenize the choice of the level of targeting by considering that firm 2 has an array of specialized advertising media available, $z \in\left\{z_{0}, z_{1}, z_{2}, \ldots, z_{n}\right\}$, so it can control the specialization of the targeting by choosing the value of $z$ within the interval $\left[z_{0}, z_{n}\right]$, with $z_{0}>\underline{z}$ and $z_{n}<1$. In order to find the optimal targeting strategy, it is first necessary to clarify how advertising costs vary with the specialization of the targeting, i.e. the curvature of $A_{1}(z)$ which, in turn, is determined by the way in which the cost per informed consumer varies with $z$. In the basic version of the model, we consider $A_{1}=A_{0}(1-z)$, which implies that, as $z$ changes, the cost per informed consumer remains constant. ${ }^{18}$ However, Esteban et al. $(2001,2006)$ report that a more specialized targeted campaign may result in a higher cost per informed consumer. In order to encompass this possibility, we specify $A_{1}(z)$ by means of a polynomial advertising cost function, $A_{1}=A_{0}(1-z)^{\gamma}$, with $\gamma \geq 1$. The cases of $\gamma=1$ and $\gamma>1$ yield,

\footnotetext{
18 Note that the cost per informed consumer is $\frac{A(z)}{N(1-z)}$, where $N$ represents the mass of potential consumers which, for the sake of simplicity, we have normalized to 1 .
} 
Table 2 Optimal targeting strategy $(\gamma=1)$

\begin{tabular}{llllllll}
\hline$z$ & $\theta^{0}(z)$ & $\alpha$ & $\beta$ & $E p_{1}$ & $E p_{2}$ & $\Pi_{1}$ & $\Pi_{2}$ \\
\hline 0.10 & 0.358 & 0.0772 & 0.704 & 141.93 & 215.55 & 22.05 & 127.43 \\
0.20 & 0.353 & 0.0774 & 0.352 & 142.04 & 215.60 & 22.07 & 127.46 \\
0.34 & 0.348 & 0.07773 & 0.207 & 142.20 & 215.66 & 22.09 & 127.50 \\
0.35 & 0.3476 & 0.07771 & 0.201 & 142.28 & 215.86 & 22.16 & 127.57 \\
0.36 & 0.3472 & 0.0770 & 0.197 & 142.33 & 216.59 & 22.42 & 127.76 \\
0.37 & 0.3468 & 0.0755 & 0.193 & 141.91 & 217.12 & 22.61 & 127.82 \\
0.38 & 0.3464 & 0.0732 & 0.188 & 141.06 & 217.45 & 22.73 & 127.75 \\
0.45 & 0.3434 & 0.037 & 0.154 & 123.62 & 214.47 & 21.66 & 124.06 \\
0.49 & 0.3418 & 0.0011 & 0.135 & 105.04 & 209.07 & 19.76 & 119.79 \\
$0.50-0.90$ & & 0 & 0 & 100.00 & 200.00 & 16.67 & 116.67 \\
\hline
\end{tabular}

Table 3 Optimal targeting strategy $(\gamma=1.5)$

\begin{tabular}{llllllll}
\hline$z$ & $\theta^{0}(z)$ & $\alpha$ & $\beta$ & $p_{1}^{e}$ & $p_{2}^{e}$ & $\Pi_{1}$ & $\Pi_{2}$ \\
\hline 0.10 & 0.3695 & 0.1091 & 0.751 & 159.23 & 222.67 & 24.65 & 132.39 \\
0.20 & 0.3627 & 0.1069 & 0.373 & 158.00 & 222.15 & 24.46 & 132.03 \\
0.35 & 0.3529 & 0.1032 & 0.212 & 156.00 & 221.31 & 24.14 & 131.44 \\
0.36 & 0.3523 & 0.1026 & 0.207 & 156.01 & 221.86 & 24.35 & 131.58 \\
0.37 & 0.3517 & 0.1013 & 0.202 & 155.69 & 222.47 & 24.57 & 131.67 \\
0.38 & 0.3511 & 0.099 & 0.198 & 154.95 & 222.87 & 24.72 & 131.63 \\
0.45 & 0.3468 & 0.064 & 0.163 & 138.49 & 220.26 & 23.76 & 128.02 \\
0.49 & 0.3444 & 0.029 & 0.142 & 120.65 & 214.92 & 21.82 & 123.70 \\
$0.50-0.90$ & & 0 & 0 & 100.00 & 200.00 & 16.67 & 116.67 \\
\hline
\end{tabular}

respectively, a linear and convex advertising cost function which, in turn, result in a constant or a higher cost per informed consumer when $z$ increases. ${ }^{19}$ In Tables 2 and 3 , we compute the model for $\left[v, q_{1}, \bar{q}_{2}\right]=[550,137.5,550]$, with an intermediate level of product differentiation, $\Delta q=300$, and $A_{0}=\frac{\Delta q}{18}$. Further, we consider a wide set of targeting possibilities, $z \in[0.1,0.11,0.12,0.13, \ldots, 0.88,0.89,0.90]$, with $z_{0}=0.1>\underline{z}=0.07$, so, if $z \leq \theta^{0}(z)$, targeting always induces firm 1 to randomly monopolize the captive market. In this setup, we study how the changes in the level of specialization of the targeting, $z$, affect $(\alpha, \beta),\left(E p_{1}, E p_{2}\right)$ and the firms' profits, $\left(\Pi_{1}, \Pi_{2}\right)$, when $\gamma=1$ and $\gamma>1$, respectively.

In order to understand the intuition behind the choice of the optimal targeting strategy, we begin by discussing how a change in $z$ affects $\alpha, \beta$ and firm 2's profits. When $z \leq \theta^{0}(z)$, an increase in $z$ generates two effects. (1) First, a higher $z$ increases the

19 The case $\gamma<1$, i.e. a decreasing cost per informed consumer, yields, qualitatively, the same results as $\gamma=1$ and, therefore, we ignore it. 
size of $[0, z]$, that is, the market which, with probability $\alpha$, firm 1 monopolizes, so we can expect firm 2 to have a higher incentive to compete for $[0, z]$, i.e. $\beta$ decreases.

(2) Secondly, a higher $z$ also has a direct impact on $\Pi_{2}(z)$. In particular, as $z$ increases, we have that firm 2 can capture a lower demand when $p_{1}=p^{M}$ (see (5)), which reduces $\Pi_{2}(z)$, and that the cost of the specialized advertising campaign decreases, which increases $\Pi_{2}(z)$. The final impact of a higher $z$ on $\Pi_{2}(z)$ turns out to depend on the properties of the targeting technology:

(a) If $\gamma=1$, an increase in $z$ leaves the advertising cost per informed consumer unchanged and we find that $\Pi_{2}(z)$ rises. In this case, we can expect that firm 1 will have a higher incentive to monopolize the captive market, i.e. $\alpha$ rises.

(b) By contrast, if $\gamma>1$, a higher $z$ increases the advertising cost per informed consumer and $\Pi_{2}(z)$ decreases, so firm 1 will have a lower incentive to monopolize the captive market, i.e. $\alpha$ falls. This explains why, in Table 2, we observe that, when $z \leq \theta^{0}(z)$, a higher $z$ yields a lower $\beta$ and a higher $\alpha$. In this case, the higher probability of monopolizing $[0, z]$ softens price competition between the firms, so profits are positively correlated with $z$. By contrast, when $\gamma>1$, Table 3 indicates that, a higher $z$ decreases both $\beta$ and $\alpha$, so firms compete more aggressively in prices, thus yielding lower profits.

Having discussed how a change in $z$ affects profits when $z \leq \theta^{0}(z)$, we now focus on the case where $z>\theta^{0}(z)$. We first note that the switch from $z \leq \theta^{0}(z)$ to $z>\theta^{0}(z)$ generates a new first-order effect on $p_{2}(z)$, which yields a higher $p_{1}^{1}, p_{2}^{1}(0)$ and, therefore, higher profits for both firms. However, we find that, when $z>\theta^{0}(z)$, profits are positively correlated with $z$ only if $z$ is sufficiently close to $\theta^{0}(z)$. The reason is that, given $t_{2}=z>\theta^{0}(z)$, as $z$ rises, firm 2 can progressively reach a smaller fraction of its potential market, $\left[\theta^{0}(z), 1\right]$, so $\Pi_{2}(z)$ falls and, once again, firm 1 will have a lower incentive to monopolize the captive market, i.e. $\alpha$ falls. This implies that a sufficiently large value of $\left(z-\theta^{0}(z)\right)$ triggers stronger price competition, thus lowering the firms' profits.

From this discussion, it follows that firm 2's profit reaches a local maximum at $z=\widehat{z}>\theta^{0}(z)$ (for example, in Tables 2 and $3 \widehat{z}=0.37$ ) and the issue is whether this solution is also a global maximum. We find that the answer to this question depends on the properties of the targeting technology. When $\gamma=1$, firm 2's profit increases for all $z \in[0.1,0.37]$, so this seller finds it optimal to choose a highly specialized targeting technology, $z^{*}=\widehat{z}=0.37$, which concentrates the ads on the set of buyers. However, when $\gamma>1$, the profit is non-monotonic in the support $z \in[0.1,0.37]$ and Table 3 shows that the local maximum, $z=\widehat{z}$, is not a global maximum. In this case, the game has a corner solution where firm 2 chooses the lowest degree of targeting specialization, provided that this targeting induces firm 1 to randomly monopolize its captive market, i.e. $z^{*}=0.10<\theta^{0}(z)$. This occurs because, when $\gamma>1$, any $z>0.10$ triggers stronger price competition, so firm 2 prefers to use a low specialized targeting. Finally, we note that, if $z \geq 0.5$, both firms find it optimal to use only uniform advertising and to charge the full information prices, i.e. $\bar{z}=0.5$.

In summary, we conclude that, if $\bar{z}>z>\underline{z}$, the use of targeted advertising allows firms to charge higher prices and, therefore, to obtain higher profits. However, in order to soften price competition, firms may find it optimal to choose either a low or a highly 
specialized targeting technology, depending on the curvature of the advertising cost function.

\section{Conclusions}

This paper studies a vertically differentiated market in which two firms compete in prices and can inform consumers about the existence of the goods, the price and the product characteristics by using either uniform advertising, which reaches the whole potential market, or specialized advertising, which targets the ads on a fragment of high valuation consumers. We show that, under reasonable market conditions, the game has a mixed strategy Nash equilibrium in which there is a positive probability that the low-quality firm behaves as a pure monopoly, which changes the pattern of price competition between the firms. In particular, we prove that the transition from uniform to targeted advertising always leads both firms to charge higher prices, and the increase in prices is more intense in markets with a low differentiation. Moreover, the combination of informational and vertical differentiation in the model yields some interesting results which contrast with the predictions of standard vertical differentiation models. For instance, we find that, for low degrees of product differentiation, the price of the low-quality firm decreases with the degree of product differentiation, and that the low-quality product may be sold at a higher expected price than the highquality product. Finally, we show that a progressive growth of specialized advertising media leads to a further increase in prices.

All these results help us to understand how the proliferation of new information technologies can affect firms and consumers. With respect to firms, the recent sharp change in direction taken by advertising expenses towards specialized advertising can be explained not only by its higher cost efficiency, but also by the fact that these new technologies allow sellers to achieve a greater degree of monopoly power, especially in highly competitive (low differentiated) markets. However, we show that firms may not be willing to use highly precise targeting so as to avoid more intense price competition. So far as consumers are concerned, our work suggests that, with targeted advertising, they will end up paying higher prices, and that the increase in price is inversely related to the cost of these media. Further, the lack of information may lead buyers to undertake inefficient purchases, in the sense that they pay a higher price for a lower quality product.

Finally, we must note that our paper constitutes a first contribution to the study of the effects of targeted advertising on the functioning of vertically differentiated markets. Some interesting aspects of this issue, such as how targeting affects product design (optimal supply of quality), still have to be explored.

\section{Appendix}

Proof of Lemma 1 (i) If $t_{1}=t_{2}=0$ and $v>\frac{\Delta q}{3}$, in equilibrium, the market is covered and profit maximization yields the reaction functions: ${ }^{20}$

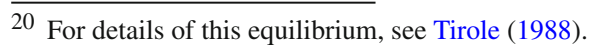




$$
\begin{aligned}
& p_{1}^{m}\left(p_{2}\right)=\frac{p_{2}}{2}, \\
& p_{2}^{m}\left(p_{1}\right)=\frac{p_{1}+\Delta q}{2} .
\end{aligned}
$$

The intersection of (7) and (8) yields the unique Nash equilibrium of the pricing game: $p_{1}^{m}=\frac{\Delta q}{3}, p_{2}^{m}=\frac{2 \Delta q}{3}$, with profits $\Pi_{1}^{m}=\frac{\Delta q}{9}-A_{0}$, and $\Pi_{2}^{m}=$ $\frac{4 \Delta q}{9}-A_{0}$. Next, let us assume that firms can target their ads on $z \leq \theta^{m}=$ $\frac{p_{2}^{m}-p_{1}^{m}}{\Delta q}$. Then, given $\left(t_{1}=0, p_{1}^{m}\right)$, firm 2 's profits under targeted advertising are: $\Pi_{2}\left(p_{2}, z\right)=p_{2} \operatorname{Min}\left\{1-\frac{p_{2}-p_{1}^{m}}{\Delta q}, 1-z\right\}-A_{1}(z)$. Assume, for the moment, that $\operatorname{Min}\left\{1-\frac{p_{2}-p_{1}^{m}}{\Delta q}, 1-z\right\}=1-\frac{p_{2}-p_{1}^{m}}{\Delta q}$. In this case, the price that maximizes $\Pi_{2}\left(p_{2}, z\right)=p_{2}\left(1-\frac{p_{2}-p_{1}^{m}}{\Delta q}\right)-A_{1}(z)$ is $p_{2}=p_{2}^{m}$, and so it holds that $1-\frac{p_{2}^{m}-p_{1}^{m}}{\Delta q} \leq 1-z$. Finally, $\frac{\partial \Pi_{2}\left(p_{2}^{m}, z\right)}{\partial z}=-A_{1}^{\prime}(z)>0$ which implies that, given the pricing-advertising strategy of firm 1, firm 2's best response is $t_{2}=z$. Therefore, $t_{1}=t_{2}=0$ cannot be part of an equilibrium.

Proof of Lemma 2 From Lemma 1 it follows that, when $\left(t_{1}=0, t_{2}=z\right)$ and both firms compete for the fully informed consumers in $[z, 1]$, the optimal prices are $\left(p_{1}^{m}, p_{2}^{m}\right)$. However, this is not an equilibrium strategy given that, if $t_{2}=z \longrightarrow \theta^{m}$ and $p_{2}=p_{2}^{m}$, then it is clear that firm 1 's best response will be to monopolize the captive market by setting $p_{1}^{M}=\operatorname{Max}\left[v, \frac{v+z q_{1}}{2}\right]>p_{1}^{m}$, in such a way that $\theta^{M}=\frac{p_{2}^{m}-p_{1}^{M}}{\Delta q}<\theta^{m}$ and the demand served by firm 1 is $D_{1}^{M}=\operatorname{Min}\left[z, \frac{v+z q_{1}}{2 q_{1}}\right]$. As a result of this monopolization strategy, if $t_{2}=z$, firm 2 faces a demand: $D_{2}=\operatorname{Min}\left[1-z, 1-\frac{p_{2}-p_{1}^{M}}{\Delta q}\right]$. Given that from (8) we know that $\frac{\partial p_{2}}{\partial p_{1}}<1$, the solution of $\operatorname{Max}_{p_{2}} p_{2}\left(1-\frac{p_{2}-p_{1}^{M}}{\Delta q}\right)-A_{1}$ yields $\frac{p_{2}-p_{1}^{M}}{\Delta q}<\theta^{m}$. Therefore, $D_{2}=1-z$, and firm 2 will respond to $p_{1}^{M}$ by charging the maximum price that the marginal consumer, $z \longrightarrow \theta^{m}$, is willing to pay, i.e. $p_{2}^{\prime}=p_{1}^{M}+\Delta q \theta^{m}$, in such a way that $\frac{p_{2}^{\prime}-p_{1}^{M}}{\Delta q}=\theta^{m}$. Given $t_{2}=z$ and $p_{2}^{\prime}$, firm 1 can either monopolize $[0, z]$, which yields a benefit $\Pi_{1}^{M}=p_{1}^{M} D_{1}^{M}-A_{0}$, or compete for $[z, 1]$, which implies maximizing $\Pi_{1}^{\prime}=p_{1}\left(\frac{p_{2}^{\prime}-p_{1}}{\Delta q}\right)-A_{0}$. The solution of this problem yields $p_{1}^{\prime}=\frac{p_{2}^{\prime}}{2}$ and profits $\Pi_{1}^{\prime}=\frac{\left[p_{1}^{M}+z \Delta q\right]^{2}}{4 \Delta q}-A_{0}$. Some calculations yield that $\Pi_{1}^{M}>\Pi_{1}^{\prime}$ implies $\left(p_{1}^{M}\right)^{2}+z^{2} \Delta q^{2}+p_{1}^{M} \Delta q\left[2 z-4 D_{1}^{M}\right]<0$. Taking into consideration that $D_{1}^{M} \leq z$, we have that $2 z-4 D_{1}^{M} \geq-2 z$, and so

$$
\begin{aligned}
0 & >\left(p_{1}^{M}\right)^{2}+z^{2} \Delta q^{2}+p_{1}^{M} \Delta q\left[2 z-4 D_{1}^{M}\right] \\
& \geq\left(p_{1}^{M}\right)^{2}+z^{2} \Delta q^{2}-2 z p_{1}^{M} \Delta q=\left[p_{1}^{M}-z \Delta q\right]^{2},
\end{aligned}
$$

which constitutes a contradiction. This shows that, when $z \longrightarrow \theta^{m}$, firm 1's best response to $\left(t_{2}=z, p_{2}^{\prime}\right)$ is to compete for $[z, 1]$. However, if both firms compete for 
the fully informed consumers in $[z, 1]$, the unique Nash equilibrium of the pricing game is $\left(p_{1}^{m}, p_{2}^{m}\right)$ which, as we have already shown, cannot be part of an equilibrium.

Proof of Proposition 1 The existence of such an equilibrium is proved by construction. We are looking for a mixed strategy equilibrium in which firm 1 charges a finite number of prices, so the equilibrium can be obtained from a set of equations which satisfy two conditions: (1) the profit function must be maximized at each point of the support of the price distribution, and (2) the profits of all the points of the support of the price distribution have to be the same. Further, we need to verify that neither firm has a profitable deviation from the strategies prescribed.

We begin by characterizing firm 2's optimal pure pricing strategies:

(i) Given the strategy profile $s_{1}=\left(p_{1}, t_{1}=0\right)$ described in Proposition 1 , we must check that the pricing strategies are optimal for the two possible advertising strategies, $t_{2}=z$ and $t_{2}=0$, and that firm 2 finds it optimal to compete when facing a low price, $p_{1}=p_{1}^{0}$.

(i1) $t_{2}=z$. In this case firm 2's expected profit is:

$$
\begin{aligned}
E \Pi_{2}= & p_{2}(z)\left\{\alpha \operatorname{Min}\left[(1-z) ;\left(1-\frac{p_{2}(z)-p_{1}^{0}}{\Delta q}\right)\right]\right. \\
& \left.+(1-\alpha) \operatorname{Min}\left[(1-z) ;\left(1-\frac{p_{2}(z)-p_{1}^{0}}{\Delta q}\right)\right]\right\}-A_{1} .
\end{aligned}
$$

Assuming that $v$ is high, i.e. $v>z q_{1}$, so that $p_{1}^{M}=v$, we can expect that, under reasonable market conditions, firm 1's monopoly price will be higher than the price set by firm $2, p_{1}^{M}=v>p_{2}(z)$. Further, under the condition

$$
\frac{p_{2}(z)-p_{1}^{0}}{\Delta q} \geq z
$$

if firm 2 competes for the marginal fully informed consumer when $p_{1}=p_{1}^{0}$, the firm faces the following problem:

$$
\operatorname{Max}_{p_{2}} E \Pi_{2}=p_{2}(z)\left[\alpha(1-z)+(1-\alpha)\left(1-\frac{p_{2}(z)-p_{1}^{0}}{\Delta q}\right)\right]-A_{1}
$$

which yields:

$$
p_{2}(z)=\frac{(1-\alpha) p_{1}^{0}+(1-\alpha z) \Delta q}{2(1-\alpha)},
$$

and the corresponding maximum expected profit $E \Pi_{2}(z)$. Firm 2 can deviate from $p_{2}(z)$ by renouncing to compete with firm 1 when this firm sets $p_{1}=p_{1}^{0}$. 
Under this deviation strategy, when $p_{1}=v$ firm 2's residual demand can be obtained by locating the indifferent consumer:

$$
\begin{aligned}
v+\theta q_{1}-p_{1}^{M} & =v+\theta q_{2}-p_{2} \Longrightarrow \theta=\frac{p_{2}-v}{\Delta q} \Longrightarrow D_{2} \\
& =\operatorname{Min}\left[1-z ; 1-\frac{p_{2}-v}{\Delta q}\right] .
\end{aligned}
$$

Accordingly, the minimum price charged by firm 2 is $p_{2}^{d}=v+z \Delta q$, and straightforward computations yield that when $D_{2}=1-\frac{p_{2}-v}{\Delta q}$ the optimal deviation price is $p_{2}^{d}=\frac{v+\Delta q}{2}$, and the demand served is $D_{2}^{d}=\frac{v+\Delta q}{2 \Delta q}$. Assuming, once again, a high $v$, i.e. $v>\Delta q(1-2 z)$, we have that

$$
D_{2}^{d}=\operatorname{Min}\left[1-z ; \frac{v+\Delta q}{2 \Delta q}\right]=1-z
$$

Note that, given $v>\frac{\Delta q}{3}$, this conditions always holds in the neighborhood of $z=\frac{1}{3}$. Thus, the maximum expected deviation profit is:

$$
\begin{aligned}
& E \Pi_{2}^{d}(z)=(v+z \Delta q) \\
& \quad \times\left\{\alpha(1-z)+(1-\alpha) \operatorname{Max}\left[0,1-\frac{(v+z \Delta q)-p_{1}^{0}}{\Delta q}\right]\right\}-A_{1} .
\end{aligned}
$$

Therefore, $p_{2}(z)$ will be the optimal price when $t_{2}=z$ if:

$$
E \Pi_{2}(z)>E \Pi_{2}^{d}(z)
$$

(i2) $t_{2}=0$. Under the competitive strategy, if $p_{1}^{M}=v>p_{2}(0)$, and the following condition holds

$$
\frac{p_{2}(0)-p_{1}^{0}}{\Delta q} \geq 0
$$

firm 2 faces the following problem:

$$
\operatorname{Max}_{p_{2}} E \Pi_{2}=p_{2}(0)\left[\alpha+(1-\alpha)\left(1-\frac{p_{2}(0)-p_{1}^{0}}{\Delta q}\right)\right]-A_{0}
$$

which yields

$$
p_{2}(0)=\frac{p_{1}^{0}(1-\alpha)+\Delta q}{2(1-\alpha)}>p_{2}(z)
$$


and the corresponding maximum expected profit $E \Pi_{2}(0)$. If firm 2 deviates by renouncing to compete with firm 1 when this firm sets $p_{1}=p_{1}^{0}$, the residual demand when $p_{1}=v$ is $D_{2}^{d}=\operatorname{Min}\left[1 ; 1-\frac{p_{2}-v}{\Delta q}\right]$, the optimal price is $p_{2}^{d}=\operatorname{Max}\left[v ; \frac{v+\Delta q}{2}\right]$ and, therefore, the maximum deviation profit is:

$$
E \Pi_{2}^{d}(0)=p_{2}^{d}\left\{\alpha D_{2}^{d}+(1-\alpha) \operatorname{Max}\left[0,1-\frac{p_{2}^{d}-p_{1}^{0}}{\Delta q}\right]\right\}-A_{0} .
$$

Accordingly, $p_{2}(0)$ will be the optimal price when $t_{2}=0$ if $E \Pi_{2}(0)>E \Pi_{2}^{d}(0)$.

(ii) Regarding firm 1's strategy, and given the strategy profile $s_{2}=\left(p_{2}, t_{2}\right)$ described in Proposition 1 , if firm 1 sets $t_{1}=0$ with probability 1 , there is a probability $\beta$ that this firm has a captive market $[0, z]$, so it faces a trade-off between competing for the segment of heterogeneous consumers in $[z, 1]$ by quoting a low price, or extracting surplus from its captive segment by setting a high price. In other words, given $s_{2}$, firm 1 can choose to serve only the segment of the market $[0, z]$ and charge the monopoly price. In this case, we have that if $v>z q_{1}$,

$$
D_{1}^{M}=\operatorname{Min}\left[z ; \frac{v+z q_{1}}{2 q_{1}}\right]=z \Longrightarrow p_{1}^{M}=\operatorname{Max}\left[v, \frac{v+z q_{1}}{2}\right]=v
$$

and, given $p_{1}^{M}=v>p_{2}^{k}, k=0,1$, the firm can guarantee itself an expected profit $E \Pi_{1}^{M}=\beta v z-A_{0}$.

Next, consider that firm 1 competes for the segment $[z, 1]$, so it faces the following problem:

$$
\operatorname{Max}_{p_{1}} E \Pi_{1}=p_{1}\left\{\beta \operatorname{Max}\left[z, \frac{p_{2}(z)-p_{1}}{\Delta q}\right]+(1-\beta)\left(\frac{p_{2}(0)-p_{1}}{\Delta q}\right)\right\}-A_{0} .
$$

Under conditions (9) and (13), the solution to this problem yields the optimal price:

$$
p_{1}=\frac{(1-\beta) p_{2}(0)+\beta p_{2}(z)}{2} .
$$

The intersection between (11), (15) and (18) yields:

$$
\begin{aligned}
& p_{1}^{0}=\frac{\Delta q(1-\alpha \beta z)}{3(1-\alpha)}, p_{2}^{0}(z)=\frac{\Delta q[4-3 \alpha z-\alpha \beta z]}{6(1-\alpha)}, \\
& p_{2}^{0}(0)=\frac{\Delta q(4-\alpha \beta z)}{6(1-\alpha)}
\end{aligned}
$$

and $\theta^{0}(z)=\frac{p_{2}^{0}(z)-p_{1}^{0}}{\Delta q}=\frac{2}{6-\alpha(3+\beta)}$. In order to ensure that these are indeed the equilibrium prices, we must check the fulfillment of conditions (9) and (13). It is 
straightforward to see that, in the neighborhood of $z=\frac{1}{3}$, both conditions hold, since

$$
\theta^{0}(z)-z=\frac{2+[-6+\alpha(3+\beta)] z}{6(1-\alpha)}>0
$$

and that (9) and (13) also hold for any $z \leq \theta^{0}(z)=\frac{2}{6-\alpha(3+\beta)}$, with $\theta^{0}(z)>\frac{1}{3}$.

In a mixed strategy equilibrium, firm 1 must be indifferent between the prices which are played with positive probability, i.e.

$$
\beta v z=p_{1}^{0}\left[\beta\left(\frac{p_{2}^{0}(z)-p_{1}^{0}}{\Delta q}\right)+(1-\beta)\left(\frac{p_{2}^{0}(0)-p_{1}^{0}}{\Delta q}\right)\right]
$$

Given its rival strategy, the only possible deviation of firm 1 is to set $t_{1}=z$. In this case, firm 1 competes for fully informed consumers, so it faces the following problem:

$$
\operatorname{Max}_{p_{1}} E \Pi_{1}^{d}=p_{1}\left[\beta\left(\frac{p_{2}^{0}(z)-p_{1}}{\Delta q}-z\right)+(1-\beta)\left(\frac{p_{2}^{0}(0)-p_{1}}{\Delta q}-z\right)\right]-A_{1},
$$

which yields

$$
p_{1}^{d}=\frac{p_{2}^{0}(0)(1-\beta)+\beta p_{2}^{0}(z)-z \Delta q}{2}
$$

and the corresponding maximum profit $E \Pi_{1}^{d}\left(t_{1}=z\right)$. Therefore, the existence of an equilibrium requires that $E \Pi_{1}^{M}>E \Pi_{1}^{d}\left(t_{1}=z\right)$.

Finally, firm 2 must be indifferent between the two advertising strategies, $t_{2}=0$ and $t_{2}=z$, which are played with positive probability, so the equilibrium must satisfy the condition:

$$
\begin{aligned}
& p_{2}^{0}(z)\left[\alpha(1-z)+(1-\alpha)\left(1-\frac{p_{2}^{0}(z)-p_{1}^{0}}{\Delta q}\right)\right]-A_{1} \\
& =p_{2}^{0}(0)\left[\alpha+(1-\alpha)\left(1-\frac{p_{2}^{0}(0)-p_{1}^{0}}{\Delta q}\right)\right]-A_{0} .
\end{aligned}
$$

Solving out (21) yields:

$$
\beta(\alpha)=\frac{\alpha z \Delta q(8-3 \alpha z)-12 \Delta A(1-\alpha)}{2 \Delta q \alpha^{2} z^{2}},
$$

whereas (20) yields an expression which implicitly defines the optimal value of $\alpha$ :

$$
9 \beta(\alpha) v z(1-\alpha)^{2}-\Delta q(1-\alpha \beta(\alpha) z)^{2}=0
$$


The solution to (22) and (23) yields the equilibrium values $\left(\alpha^{0}, \beta^{0}\right)$, which can be substituted in (19) to obtain the optimal prices $p_{1}^{0}, p_{2}^{0}(z), p_{2}^{0}(0)$, and $\theta^{0}(z)=\frac{p_{2}^{0}(z)-p_{1}^{0}}{\Delta q}$.

It is straightforward to check that, given $t_{1}=0$ and firm 2's equilibrium strategy, firm 1's mixed pricing strategy can entail only a two-point distribution. To see this point, we note that firm 1's profits are:

$$
E \Pi_{1}=\operatorname{Max}\left\{\beta p_{1} \operatorname{Min}\left(z ; z-\frac{p_{1}-v}{q_{1}}\right) ; p_{1}\left(\frac{E p_{2}-p_{1}}{\Delta q}\right)\right\}-A_{0}
$$

so we consider two cases:

(a) If $\beta p_{1} \operatorname{Min}\left(z ; z-\frac{p_{1}-v}{q_{1}}\right) \geq p_{1}\left(\frac{E p_{2}-p_{1}}{\Delta q}\right)$, then $E \Pi_{1}\left(p_{1}\right)=\beta p_{1}$ $\operatorname{Min}\left(z ; z-\frac{p_{1}-v}{q_{1}}\right)$ and, according to condition (16), profit maximization yields a unique solution at $p_{1}^{M}=v$.

(b) If $p_{1}\left(\frac{E p_{2}-p_{1}}{\Delta q}\right) \geq \beta p_{1} \operatorname{Min}\left(z ; z-\frac{p_{1}-v}{q_{1}}\right)$, then $E \Pi_{1}\left(p_{1}\right)=p_{1}\left(\frac{E p_{2}-p_{1}}{\Delta q}\right)$ and, given problem (17), profit maximization yields a unique solution at $p_{1}=p_{1}^{0}$.

Further, (20) implies that charging $p_{1}=v$ and $p_{1}=p_{1}^{0}$ yields the same profit. Thus, we have that the profit function can be maximized only by two prices, and that the profits corresponding to these two prices are the same. Therefore, the support of the price distribution of firm 1's equilibrium mixed strategy necessarily consists of only two prices, $p_{1} \in\left\{p_{1}^{0}, v\right\}$, which are played with positive probabilities.

In summary, when $z \leq \theta^{0}(z)$, an equilibrium exists if $z \leq \frac{1}{3}$, there is a parameter constellation $\left[v, \Delta q, A_{0}, A_{1}\right]$ such that $v>p_{2}^{0}(0)$ and the following non-deviation conditions hold: (i) $E \Pi_{2}(z)>E \Pi_{2}^{d}(z)$; (ii) $E \Pi_{2}(0)>E \Pi_{2}^{d}(0)$; (iii) $E \Pi_{1}^{M}>E \Pi_{1}^{d}\left(t_{1}=z\right)$. Computing the model the following market scenario, $\left[v, z, q_{1}, \bar{q}_{2}, A_{0}, A_{1}\right]=[550,0.25,137.5,550,15.27,11.45]$, we find that the equilibrium exists for $\Delta q \in[146.6,412.5]$.

Proof of Proposition 2 The pattern of competition between firms when $z>\theta^{0}(z)$ is very similar to the case where $z \leq \theta^{0}(z)$ so, in what follows, we will only highlight the differences from the proof of Proposition 1. Regarding firm 2's optimal strategy when $t_{2}=z$, we note that restriction (9) now becomes $\frac{p_{2}(z)-p_{1}^{1}}{\Delta q}<z$. Under this condition, firm 2 faces a demand:

$$
D_{2}=\left[\alpha(1-z)+(1-\alpha) \operatorname{Min}\left\{(1-z) ;\left(1-\frac{p_{2}-p_{1}^{1}}{\Delta q}\right)\right\}\right]=1-z
$$

This implies that there is a first-order effect on the price, that is, the optimal price leaves the marginal consumer, $\theta=z$, indifferent between buying the two products:

$$
\theta^{1}(z)=\frac{p_{2}(z)-p_{1}^{1}}{\Delta q}=z \Longrightarrow p_{2}^{1}(z)=p_{1}^{1}+z \Delta q
$$


This pricing strategy generates an equilibrium profit of $E \Pi_{2}(z)=\left(p_{1}^{1}+z \Delta q\right)(1-$ $z)-A_{1}$, and firm 2 will not deviate if (12) holds. The solutions to firm 1's problem, firm 2's problem when $t_{2}=0$, and the corresponding deviation strategies, are identical to those described in Proposition 1. Therefore, the intersection between (15), (18) and (24) yields the optimal prices, $p_{1}^{1}, p_{2}^{1}(0)$ and $p_{2}^{1}(z)$, whilst resolving the following two equations:

$$
\begin{aligned}
& \beta v z=p_{1}^{1}\left[\beta\left(\frac{p_{2}^{1}(z)-p_{1}^{1}}{\Delta q}\right)+(1-\beta)\left(\frac{p_{2}^{1}(0)-p_{1}^{1}}{\Delta q}\right)\right], \\
& \left(p_{1}^{1}+z \Delta q\right)(1-z)-A_{1}=p_{2}^{1}(0)\left[\alpha+(1-\alpha)\left(1-\frac{p_{2}^{1}(0)-p_{1}^{1}}{\Delta q}\right)\right]-A_{0}
\end{aligned}
$$

yields the equilibrium values $\left(\alpha^{1}, \beta^{1}\right)$. Finally, from Proposition 1 , we have that the condition $z>\theta^{0}(z)$ implies $z>\frac{1}{3}$. In sum, an equilibrium exists if $z>\frac{1}{3}$ and there is a parameter constellation $\left[v, \Delta q, A_{0}, A_{1}\right]$ such that $v>p_{2}^{1}(0), v>p_{2}^{1}(z)$ and the following non-deviation conditions hold: (i) $E \Pi_{2}(z)>E \Pi_{2}^{d}(z)$; (ii) $E \Pi_{2}(0)>$ $E \Pi_{2}^{d}(0)$; (iii) $E \Pi_{1}^{M}>E \Pi_{1}^{d}\left(t_{1}=z\right)$. Computing the model for the following market scenario, $\left[v, z, q_{1}, \bar{q}_{2}, A_{0}, A_{1}\right]=[550,0.45,137.5,550,15.27,8.40]$, we find that the equilibrium exists for $\Delta q \in[137.5,412.5]$.

Proof of Proposition 3 (i) Case 1: $z \leq \theta^{0}(z)$. We first note that $\left(p_{1}^{m}, p_{2}^{m}\right)$ are defined by the following set of first order conditions (FOCs):

$$
\begin{gathered}
p_{2}^{m}-2 p_{1}^{m}=0, \\
\Delta q+p_{1}^{m}-2 p_{2}^{m}=0,
\end{gathered}
$$

whereas the FOCs which define $\left(p_{1}^{0}, p_{2}^{0}(z), p_{2}^{0}(0)\right)$ are, respectively:

$$
\begin{gathered}
(1-\beta) p_{2}(0)+\beta p_{2}(z)-2 p_{1}^{0}=0, \\
\Delta q(1-\alpha z)+p_{1}^{0}(1-\alpha)-2 p_{2}(z)(1-\alpha)=0, \\
\Delta q+p_{1}^{0}(1-\alpha)-2 p_{2}(0)(1-\alpha)=0 .
\end{gathered}
$$

From (29) and (30) we have that

$$
\begin{aligned}
& \Delta q(1-\alpha z)-2 p_{2}(z)(1-\alpha)=\Delta q-2 p_{2}(0)(1-\alpha) \\
& \Longrightarrow p_{2}(0)=p_{2}(z)+\frac{\Delta q \alpha z}{2(1-\alpha)}
\end{aligned}
$$


so $p_{2}^{0}(0)>p_{2}^{0}(z)$. Further, from (28) and (26) we observe that:

$$
\begin{gathered}
p_{1}^{0}=\frac{(1-\beta) p_{2}(0)+\beta p_{2}(z)}{2}=\frac{E p_{2}}{2}, \\
p_{1}^{m}=\frac{p_{2}^{m}}{2}=\frac{E p_{2}}{2},
\end{gathered}
$$

whereas (26) and (27) imply that $p_{2}^{m}$ is determined by:

$$
\Delta q+\frac{p_{2}^{m}}{2}-2 p_{2}^{m}=0
$$

We now operate in the FOC corresponding to $p_{2}(z)$, i.e. in (29), making use of (31) and (32):

$$
\begin{aligned}
& \frac{\partial \Pi}{\partial p_{2}(z)}=\Delta q(1-\alpha z)+(1-\alpha)\left[\frac{(1-\beta)\left(p_{2}(z)+\frac{\Delta q \alpha z}{2(1-\alpha)}\right)+\beta p_{2}(z)}{2}\right] \\
& -2 p_{2}(z)(1-\alpha)=\Delta q(1-\alpha z)+(1-\alpha)\left[\frac{p_{2}(z)}{2}+\frac{(1-\beta) \Delta q \alpha z}{4(1-\alpha)}-2 p_{2}(z)\right] .
\end{aligned}
$$

Next, we evaluate this expression in $p_{2}(z)=p_{2}^{m}$ and operate making use of (34):

$$
{\frac{\partial \Pi_{2}}{\partial p_{2}(z)}}_{\mid p_{2}(z)=\frac{2 \Delta q}{3}}=\Delta q\left[\alpha(1-z)+\frac{(1-\beta) \alpha z}{4}\right]>0,
$$

which proves that $p_{2}(z)>p_{2}^{m}$ and so, given (31), that $p_{2}(0)>p_{2}^{m}$. Finally, from this it follows that $E p_{2}=\beta p_{2}(z)+(1-\beta) p_{2}(0)>p_{2}^{m}$ which, given (32) and (33), implies $p_{1}^{0}>p_{1}^{m}$.

(ii) Case 2: $z>\theta^{0}(z)$. In this case, $\left(p_{1}, p_{2}(z), p_{2}(0)\right)$ are defined by (28), (30) and $p_{2}(z)-p_{1}^{1}-z \Delta q=0$. From these equations, we have that $p_{1}^{1}=\frac{(1-\beta) p_{2}(0)+\beta z \Delta q}{2-\beta}$, so

$$
\frac{\partial \Pi_{2}}{\partial p_{2}(0)}=\Delta q+\left(\frac{(1-\beta) p_{2}(0)+\beta z \Delta q}{2-\beta}\right)(1-\alpha)-2 p_{2}(0)(1-\alpha) .
$$

Next, considering $z>\frac{1}{3}$, we evaluate this expression in $p_{2}(0)=p_{2}^{m}=\frac{2 \Delta q}{3}$, obtaining

$$
{\frac{\partial \Pi_{2}}{\partial p_{2}(0)}}_{\mid p_{2}(0)=\frac{2 \Delta q}{3}}>\Delta q\left(1+(1-\alpha)\left(\frac{3 \beta-6}{3(2-\beta)}\right)\right)>0 \Longrightarrow p_{2}(0)>p_{2}^{m} \text {. }
$$

In order to prove $p_{1}^{1}>p_{1}^{m}$, we have that

$$
\frac{\partial \Pi_{1}}{\partial p_{1}^{1}}=(1-\beta)\left(\frac{\Delta q+(1-\alpha) p_{1}^{1}}{2(1-\alpha)}\right)+\beta p_{1}^{1}+\beta z \Delta q-2 p_{1}^{1}
$$


If we evaluate this expression in $p_{1}^{1}=p_{1}^{m}$, taking into account that $z>\frac{1}{3}$, we obtain:

$$
{\frac{\partial \Pi_{1}}{\partial p_{1}^{1} \mid p_{1}^{1}=\frac{\Delta q}{3}}}=\frac{\Delta q}{6(1-\alpha)}[(1-\alpha)(6 \beta z+\beta-3)+3(1-\beta)]>0 \Longrightarrow p_{1}^{1}>p_{1}^{m} \text {. }
$$

Finally, $p_{2}(z)=p_{1}^{1}+z \Delta q>p_{1}^{m}+z \Delta q>\frac{2 \Delta q}{3}=p_{2}^{m}$.

Open Access This article is distributed under the terms of the Creative Commons Attribution Noncommercial License which permits any noncommercial use, distribution, and reproduction in any medium, provided the original author(s) and source are credited.

\section{References}

Esteban L, Gil A, Hernández JM (2001) Informative advertising and optimal targeting in a monopoly. J Ind Econ 49:161-180

Esteban L, Gil A, Hernández JM (2004) Pricing with endogenous direct advertising in a monopoly. Rev Ind Organ 25:129-154

Esteban L, Hernández JM, Moraga-Gonzalez JL (2006) Customer directed advertising and product quality. J Econ Manag Strategy 15(4):943-968

Esteban L, Hernández JM (2007) Strategic targeted advertising and market fragmentation. Econ Bull 12: $1-12$

Faulds DJ, Lonial SC (2001) Price-quality relationship of nondurable consumer products: a European and United States perspective. J Econ Soc Res 3(1):59-76

Galeotti A, Moraga-Gonzalez JL (2008) Segmentation, advertising and prices. Int J Ind Organ 26: 1106-1119

Grossman G, Shapiro C (1984) Informative advertising with differentiated products. Rev Econ Stud 51: 63-82

Iyer G, Soberman D, Miguel Villas-Boas JM (2005) The targeting of advertising. Mark Sci 24:461-473

Kerin RA, Hartley SW, Rudelius (2009) Marketing. MacGraw-Hill/Irvin

Kotler P, Armstrong G (1998, 2007) Marketing. An introduction. Prentice Hall, Englewood Cliffs

Narasimhan C (1988) Competitive promotional strategies. J Bus 61:427-449

Roy S (2000) Strategic segmentation of a market. Int J Ind Organ 18(8):1279-1290

Shaked A, Sutton J (1982) Relaxing price competition through product differentiation. Rev Econ Stud 49:3-13

Stahl DO (1994) Oligopolistic pricing and advertising. J Econ Theory 64:162-177

Steenkamp J-BEM (1988) The relationship between price and quality in the marketplace. De Economist 136(4):491-507

Stegeman M (1991) Advertising in competitive markets. Am Econ Rev 81:210-223

Tirole J (1988) The theory of industrial organization. MIT Press, Cambridge 